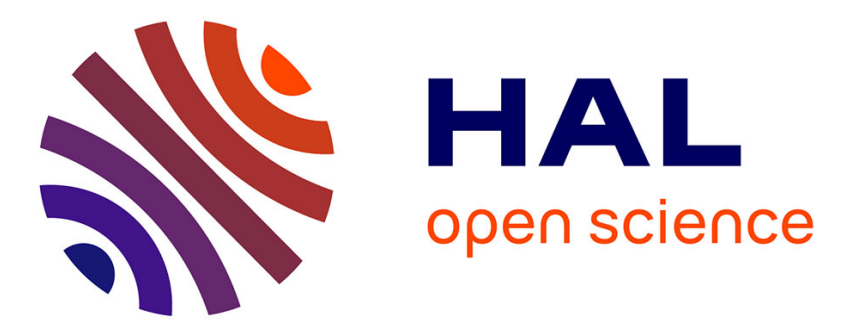

\title{
Les enjeux du leadership au Conseil de sécurité : responsabiliser ou contrôler les opérations de paix de l'ONU?
}

\author{
David Ambrosetti, Mélanie Cathelin
}

\section{- To cite this version:}

David Ambrosetti, Mélanie Cathelin. Les enjeux du leadership au Conseil de sécurité : responsabiliser ou contrôler les opérations de paix de l'ONU ?. La revue internationale et stratégique, 2007, 68 (4), pp.69-79. 10.3917/ris.068.0069 . halshs-02293613

\section{HAL Id: halshs-02293613 \\ https://shs.hal.science/halshs-02293613}

Submitted on 20 Sep 2019

HAL is a multi-disciplinary open access archive for the deposit and dissemination of scientific research documents, whether they are published or not. The documents may come from teaching and research institutions in France or abroad, or from public or private research centers.
L'archive ouverte pluridisciplinaire HAL, est destinée au dépôt et à la diffusion de documents scientifiques de niveau recherche, publiés ou non, émanant des établissements d'enseignement et de recherche français ou étrangers, des laboratoires publics ou privés. 


\section{Les enjeux du leadership au Conseil de sécurité : responsabiliser ou contrôler les opérations de paix de l'ONU?}

/ David Ambrosetti est docteur en science politique, chercheur au CNRS (Institut des Sciences sociales du Politique, Université Paris X-Nanterre). Il consacre ses travaux à la prise de décision au Conseil de sécurité au cœur des conflits armés

/ Mélanie Cathelin est doctorante en science politique au Centre d'études d'Afrique noire (CEAN) de l'IEP de Bordeaux. Elle est spécialisée sur la politique canadienne de gestion des conflits en Afrique

\section{Résumé}

$\mathrm{Au}$ sein du Conseil de sécurité, le leadership reconnu à un État sur un dossier est un élément essentiel pour comprendre les décisions de l'ONU en matière de gestion des conflits armés. Ce rôle social reconnu de façon informelle par les membres du Conseil cristallise les enjeux d'influence diplomatique et de crédibilité propres au travail multilatéral. La diplomatie américaine reste ici incontournable dans la désignation de la délégation leader. Mais elle a également contribué fortement au façonnement de ce rôle de leader au cours des années 1990. D'où l'apparent paradoxe : grâce à l'évolution de ce rôle, l'Administration américaine actuelle, dont le goût pour l'unilatéralisme n'est plus à démontrer, a pu accepter une expansion considérable des opérations de paix de l'ONU, en Afrique subsaharienne en particulier.

Figures emblématiques de l'action de l'ONU, les opérations de maintien de la paix font partie des pratiques de gestion des conflits les plus critiquées, pour leur inefficacité, leur lourdeur bureaucratique, ou encore leur chaîne de commandement défaillante. À ce titre, le maintien de la paix a souvent été jugé peu digne d'intérêt par les États les plus puissants. Pourtant, les opérations de paix de l'ONU connaissent aujourd'hui un développement sans précédent, avec plus de 92000 personnes sous l'autorité du Département des opérations de maintien de la paix (DOMP) et un budget annuel approchant les 5 milliards de dollars en 2006-2007 pour les opérations en cours. Les débats actuels concernant le déploiement au Darfour d'une mission «hybride» ONU/Union africaine, ou ceux relatifs à la constitution d'une FINUL renforcée au Liban au cours de l'été 2006 témoignent bien de l'enjeu de politique internationale que représente le maintien de la paix. Si cette action pacificatrice de l'ONU présente un tel enjeu international, c'est que les États, et en particulier les plus puissants d'entre eux, jouent toujours leur influence sur ces dossiers. C'est bien le fort investissement politique de ces derniers qui permet de comprendre cette évolution récente des opérations de paix.

Il semble alors paradoxal que cette expansion du maintien de la paix ait pu se produire à un moment où la première puissance mondiale s'est illustrée par un rejet ostensible des 
mécanismes de coopération multilatérale. Seule l'analyse des processus concrets aboutissant à la création de ces opérations ${ }^{1}$ permet de comprendre ce paradoxe, de voir que si le rapport de force n'est jamais absent dans le jeu multilatéral onusien, il se joue sur un registre profondément distinct d'une logique unilatérale. En effet, le travail multilatéral au sein du Conseil de sécurité de l'ONU alimente des logiques de répartition officieuse des responsabilités et des rôles, qui impliquent une reconnaissance collective de ces rôles, bien au-delà de l'exercice brut - et solitaire - de la puissance militaire. Si l'ONU peut être interprétée comme le relais des intérêts des États les plus puissants, elle ne se réduit pas à cela : les décisions multilatérales supposent invariablement des inflexions des intérêts initialement portées par les États. Ce sont les processus qui conduisent à ces inflexions et ces décisions multilatérales qu'il faut donc comprendre.

Au cœur de ces opérations de paix de l'ONU apparaît la figure pivot du «leader ». Ce terme de leader est utilisé par les diplomates eux-mêmes pour désigner une position d'influence reconnue par ses pairs à une délégation sur un dossier particulier. Cette influence se traduit notamment dans ses relations avec les services du Secrétariat des Nations unies à New York, ou encore lors de la négociation des orientations du Conseil de sécurité. L'investissement de ce rôle officieux permet également à une délégation d'obtenir de la part de ses partenaires divers soutiens matériels et diplomatiques au profit des élites politiques qu'elle soutient sur le terrain. Ici résident la portée éminemment politique du rôle de leader, et son intérêt pour l'étude de la politique internationale : à travers les règles informelles qui président à sa reconnaissance collective et à son exercice au quotidien se jouent l'influence des diplomates sur un dossier donné au sein de l'ONU mais également au cœur de la situation conflictuelle traitée. L'analyse de cette position d'influence diplomatique permet ainsi une meilleure compréhension des mécanismes de mobilisation et de prise de décision à l'ONU face aux situations de conflits armés, particulièrement africains ${ }^{2}$. Enfin, elle ouvre de nouvelles pistes d'explication sur les débats entourant l'efficacité et la crédibilité du maintien de la paix onusien dans son ensemble.

Rôle officieux et informel, le leadership n'est pas un rôle univoque, d'où la nécessité de rendre compte avant toute chose de ses différentes formes. Cette nature changeante est directement liée à l'enjeu politique du leadership. À cet égard, les choix de la diplomatie américaine doivent être particulièrement examinés, tant ils ont affecté les modalités de reconnaissance et d'exercice de ce rôle de leader, et par voie de conséquence le fonctionnement même du maintien de la paix onusien.

\section{Les formes changeantes du leadership}

Établir l'existence d'un leader au sein du Conseil de sécurité sur une question donnée requiert l'utilisation d'un faisceau d'indices tirés du fonctionnement quotidien du Conseil, dans sa dimension la plus routinière et bureaucratique. La répartition des rôles au Conseil de sécurité se manifeste en premier lieu par une répartition des tâches entre les différents

\footnotetext{
1. Cette analyse concrète apparaît peu dans la littérature sur le maintien de la paix. On trouvera néanmoins des éléments utiles dans David M. Malone (ed), The United Nations Security Council: From the Cold War to the 21st Century, Boulder-London, Lynne Rienner, 2004. Le présent article veut ainsi contribuer à une meilleure connaissance des processus onusiens, à travers l'étude des interactions entre diplomates siégeant au Conseil de sécurité.

2. Parce qu'ils représentent $75 \%$ du travail quotidien des membres du Conseil de sécurité, cet article traitera en priorité des conflits africains (Rwanda, Sierra Leone, Zaïre/RDC).
} 
acteurs. Cette division du travail dépend à la fois des préférences exprimées par les acteurs (de leurs intérêts) et des ressources matérielles et diplomatiques à leur disposition.

La prétention au leadership peut être identifiée dès la phase de l'inscription d'un nouveau dossier à l'ordre du jour du Conseil de sécurité. La délégation qui demande instamment une telle inscription au président du Conseil prétend vraisemblablement à un rôle d'influence particulier sur cette question, qui présente davantage d'enjeux pour elle que pour ses partenaires. Une fois le Conseil saisi, cette délégation joue un rôle moteur dans la tenue des discussions. Elle est la première à demander la parole au président à la table du Conseil. C'est également elle qui rédige les projets de résolution, qu'elle diffuse ensuite auprès des autres délégations membres.

Ces discussions préparatoires concernent également les relations entre le Conseil de sécurité et le Secrétariat, en particulier le Département des opérations de maintien de la paix (DOMP) lorsqu'il s'agit d'une résolution concernant directement une mission de paix sous commandement de l'ONU. Outre les considérations politiques des membres du Conseil, la délégation leader doit identifier une ligne d'action acceptable au plan opérationnel aux yeux du DOMP et des États fournisseurs de Casques bleus. Dans le cas contraire, le DOMP risque de ne pas réunir les troupes requises. Les difficultés rencontrées par le DOMP depuis août 2006 jusqu'à aujourd'hui pour la constitution d'une lourde opération de paix au Darfour, dans un contexte de vive opposition de Khartoum, en témoignent.

Lorsque la mission de paix est décidée et déployée, la délégation leader en assure le suivi en lien avec le Secrétariat des Nations unies et les autres capitales régionales et délégations membres du Conseil de sécurité intéressées par le dossier. Elle pèse sur la nomination des responsables de cette opération qui doivent avoir sa confiance. Et en cas d'urgence, de crise, elle sera celle qui œuvrera à mobiliser le Conseil de sécurité en demandant l'inscription exceptionnelle de la question à l'ordre du jour ou la tenue de conférences ou de débats publics pour maintenir l'intérêt médiatique (comme l'a fait la diplomatie française à propos de la Côte-d'Ivoire ou, très récemment, du Tchad et de la République centrafricaine). Ce faisant, cette délégation occupe sur ce dossier une position de confiance aux yeux de ses partenaires, qui d'une certaine manière lui délèguent leur propre responsabilité assumée collégialement en matière de préservation de la paix et de la sécurité internationale. Cette confiance peut aller jusqu'à déléguer à l'État leader la charge d'assurer une protection armée de l'opération de l'ONU qu'il pilote au Conseil de sécurité, via un déploiement militaire national ou multinational.

Notons ici l'écart considérable qui existe entre une prise de parole à la suite de l'adoption d'une résolution, une prise en charge de la rédaction des projets de résolution et l'envoi d'un contingent à l'appui d'une opération de paix de l'ONU. Il convient dès lors de distinguer entre différentes figures du leadership.

À une extrémité, se trouvent des délégations qui se proposent de «tenir la plume » sur un dossier particulier, c'est-à-dire d'assumer une sorte de secrétariat officieux parmi les membres du Conseil de sécurité sur une question donnée. Dans ce cas, ladite délégation entend prendre en charge une part de l'effort collectif induit par le fonctionnement quotidien du Conseil sur les dossiers qui n'ont pas trouvé preneur, rien de plus. Par exemple, peu après l'élection du Mexique comme membre non permanent en 2002, l'ambassadeur mexicain Adolfo Aguilar Zinzer se proposa d'assurer la présidence du 
comité de suivi des sanctions pour la Sierra Leone. On ne peut ici parler d'une influence prépondérante mexicaine sur le dossier du suivi des sanctions, même si une certaine expertise lui était reconnue. Cette première figure du leadership correspond donc à un degré minimal du rôle quant à sa signification et sa portée politiques, sorte de leadership par défaut.

À l'opposé de cette figure, se trouve celle renvoyant à la répartition des dossiers selon les zones d'influence prioritaire revendiquées avec succès par certains acteurs, et ce de longue date, parmi les membres permanents en particulier. Jusqu'au milieu des années 1990, toute question concernant un État africain francophone conduisait le président du Conseil à s'adresser préalablement à la délégation française, pour connaître ses préférences. Et si le Conseil se saisissait de la question (avec l'accord français ou à la demande de la délégation française), cette délégation se trouvait en position d'influence particulière. La reconnaissance de cette zone d'influence exclusive française s'est quelque peu effritée au cours des années 1990, en raison notamment des controverses suscitées par l'action de la France au Rwanda entre 1990 et 1994, mais le principe, qui s'applique à tous les membres permanents, n'a pas totalement disparu. Il est à noter que ces zones d'influence prioritaire peuvent être reconnues à des États membres qui ne siègent pas nécessairement au Conseil. Ces derniers œuvreront alors à entretenir la reconnaissance collective de leur zone d'influence au travers de démarches bilatérales auprès de délégations amies siégeant au Conseil, qui relaieront leurs préférences (ce fut le cas de l'Australie à propos du dossier du Timor oriental).

Entre ces pôles en matière d'intentionnalité et d'intensité du leadership reconnu à une délégation, les figures possibles sont nombreuses. Ainsi, les principes régissant la répartition géographique des dossiers s'appliquent également à une répartition thématique. La campagne électorale menée par la diplomatie canadienne en vue d'être élue au Conseil de sécurité à l'automne 1998 fournit ici un bon exemple de construction en amont d'un leadership thématique. Cette campagne, axée sur la promotion de la sécurité humaine et sur l'efficacité du maintien de la paix et des sanctions, a en effet permis à cet acteur de construire sa crédibilité sur ces dossiers, à travers notamment une tournée " électorale » menée par l'ambassadeur du Canada à l'ONU, Robert Fowler, auprès des capitales africaines. Le succès rencontré par cette entreprise a, par la suite, autorisé cette délégation à revendiquer - et à se voir reconnaître - un rôle de leader sur des dossiers tels que celui de la protection des civils dans les conflits armés. Lorsqu'elle présidait le Conseil, en février 1999 et en avril 2000, elle a ainsi inscrit à six reprises cette question à l'ordre du jour, et a organisé deux débats publics, donnant lieu à la production d'un rapport du Secrétaire général de l'ONU sur le sujet ${ }^{3}$ et à l'adoption de la résolution 1265 en septembre 2000, par laquelle le Conseil de sécurité affirmait sa volonté d'adopter des mesures plus efficaces et appropriées pour protéger les civils. Elle a par ailleurs été élue à la présidence du comité du Conseil de sécurité sur les sanctions en Angola, sur la base d'un agenda de réduction de l'impact des sanctions internationales sur les populations civiles, conformément à son « programme électoral».

On le voit, le rôle de leader confère à la délégation qui l'investit une position d'influence et de confiance vis-à-vis de ses pairs. Mais une telle position est fragile, car la reconnaissance collective du rôle s'opère de façon diffuse. Le leadership peut donc être perdu si les

\footnotetext{
3. Conseil de sécurité de l'ONU, Rapport du Secrétaire général au Conseil de sécurité sur la protection des civils en période de conflit armé, S/1999/957, 8 septembre 1999.
} 
comportements changent. Il s'agit dès lors d'analyser les modes de négociation de ce rôle entre les différents acteurs, et leur capacité relative à peser sur la structuration des préférences collectives du Conseil de sécurité.

\section{Les modes de négociation du leadership : le poids des préférences américaines}

La question de la crédibilité reconnue à la délégation leader ou candidate au leadership se révèle centrale dans la conquête et/ou la préservation de sa position d'influence sur un dossier. Elle est étroitement fonction de la capacité d'un acteur à gagner la confiance et le soutien de ses pairs. Mais toute marque de confiance ou de défiance n'affecte par l'avenir de ce rôle de la même manière, selon les ressources matérielles et sociales à disposition de chaque délégation. Les critères même du crédit et du discrédit sont donc en partie façonnés par les préférences des délégations les plus puissantes, États-Unis en tête.

\section{Enjeux de crédibilité entre pairs, différentiels de ressources entre rivaux}

Le rôle de leader qui était « spontanément » reconnu à la délégation française en matière de conflits armés en Afrique francophone tenait par exemple à la confiance dont elle bénéficiait de la part de ses partenaires quant à la capacité de la France d'atteindre des objectifs compatibles avec le mandat du Conseil. C'est en vertu de cette confiance que ses partenaires lui «déléguaient» la gestion de ces dossiers et pouvaient donc s'en désintéresser : Paris voulait fortement ce leadership, il avait les moyens de l'assumer, et ce dans un sens globalement conforme au mandat du Conseil, c'est-à-dire la recherche de la paix, ou de la stabilité, plus exactement. Les uns y gagnaient un confort mental dans la gestion d'un dossier qui les intéressait moyennement, les autres la possibilité de faire valoir leurs intérêts au cœur de cette gestion de conflit, c'est-à-dire de promouvoir un type de résolution du conflit au détriment d'autres envisageables ${ }^{4}$.

Cette crédibilité reconnue par ses partenaires consiste aussi dans la capacité d'être entendu auprès des acteurs politico-militaires locaux ou régionaux directement impliqués dans le conflit armé. Autrement dit, pour être crédible au sein du Conseil, il faut faire état d'une crédibilité minimale auprès de ces acteurs locaux et régionaux pour espérer peser sur leurs choix. Dans notre exemple, Paris pouvait ainsi faire jouer ses relations avec les leaders politiques africains et engager des moyens militaires et financiers suffisants pour soutenir les négociations.

Pour la délégation leader, la contrepartie de cette mobilisation de ressources propres dans la résolution d'un conflit au nom du Conseil de sécurité est claire : une fois ce leadership reconnu, elle bénéficie d'un surplus de ressources à l'appui de son action. Ces ressources sont bien sûr matérielles, telles qu'un déploiement de Casques bleus, la présence de bailleurs de fonds, d'opérateurs humanitaires et d'acteurs économiques pour la reconstruction, autant de ressources qui lui permettent de négocier plus efficacement avec les parties au conflit en leur offrant diverses incitations. Mais il s'agit également de ressources symboliques, en particulier d'une légitimation fournie par un mandat de l'ONU à l'appui des choix défendus au cœur du conflit, par exemple à l'appui de sanctions ou

\footnotetext{
${ }^{4}$. C'est ainsi que Chester A. Crocker, Fen Osler Hampson et Pamela Aall, Taming Intractable Conflicts. Mediation in the Hardest Cases, Washington, United States Institute of Peace Press, 2004, p. 24, caractérisent, à raison selon nous, l'action diplomatique au cœur des conflits armés.
} 
d'un recours au « shaming $»^{5}$ dans les couloirs diplomatiques internationaux. Ce leadership peut également servir à valoriser un gouvernement en politique intérieure, comme l'a illustré la rhétorique de Tony Blair sur les questions africaines ${ }^{6}$.

\section{Gagner la confiance américaine pour accéder au leadership}

Bien entendu, toutes les délégations membres n'ont pas les mêmes capacités de peser sur la « négociation » plus ou moins implicite du contenu du rôle, en raison de la grande diversité des ressources matérielles et institutionnelles à leur disposition. Une attention toute particulière mérite ici d'être accordée à la délégation américaine : sa position dans l'espace social du Conseil fait d'elle un élément incontournable. Cette délégation présente la spécificité de jouer comme une contrainte permissive sur le travail des autres délégations, dans la mesure où l'acceptation d'un projet de résolution en matière d'opérations de paix dépend en grande partie de l'attitude adoptée par Washington à son égard, en tant que principal contributeur du budget des opérations de paix de l'ONU (même si Washington accumule régulièrement d'importants arriérés de paiement vis-à-vis de l'ONU). La reconnaissance du rôle de leader apparaît dès lors fonction de la capacité d'une délégation à gagner la confiance de ses homologues américains.

Les jeux diplomatiques entourant la création de la force multinationale au Zaïre en novembre 1996 illustrent singulièrement bien cette idée de contrainte permissive. À cette date, en raison de l'absence de consensus entre les principaux acteurs du Conseil de sécurité sur le dossier zaïrois, le rôle de leader est laissé en vacance. Cette situation tient en grande partie à l'attitude ambiguë des diplomates américains : ils sont en effet tout à la fois contraints par l'hostilité du Congrès et de l'opinion publique américaine à toute forme d'intervention américaine sur le sol africain, et soucieux de protéger leurs intérêts dans la région. Désirant faciliter le renversement de Mobutu Sese Seko, ils s'opposent aux appels à une intervention armée internationale pouvant freiner la rébellion de Laurent-Désiré Kabila soutenue par l'Armée patriotique rwandaise du président Paul Kagamé. Confrontés à une forte mobilisation des médias occidentaux et des délégations à l'ONU, ils acceptent finalement l'idée d'une intervention multinationale mais œuvrent à discréditer tout leadership de la délégation française et à défendre l'idée d'un leadership militaire canadien soutenu militairement par Washington et relayé par la délégation américaine au Conseil de sécurité. L'appui américain, à la fois matériel et symbolique, dont bénéficient les dirigeants canadiens se révèle décisif dans la reconnaissance de leur leadership militaire au sein du Conseil, qui culmine avec l'adoption de la résolution 1080 du 15 novembre 1996, autorisant ladite force multinationale. Par la suite, la délégation américaine n'a eu de cesse de discuter point par point la viabilité de ce projet d'opération, dont elle ne voulait aucunement, et ce afin de gagner du temps et d'étouffer finalement cette mobilisation diplomatique. Cette opération multinationale sous commandement canadien au Zaïre en 1996 n'eut jamais lieu. Washington fut perçu par les diplomates et les militaires canadiens comme disposant d'un veto de fait sur le devenir de la mission. Cela les plaçait dans une position particulièrement délicate vis-à-vis de leurs pairs, dans la mesure où ils devaient sans cesse composer entre les attentes suscitées par leur projet, de la part des États

\footnotetext{
5. Le «shaming » consiste à nommer explicitement des États ou des individus auteurs de comportements contestables ou illégitimes dans une arène multilatérale donnée, par exemple dans des rapports d'experts nommés par le Secrétaire général de l'ONU, avec l'espoir de voir ces acteurs modifier leurs comportements par souci pour leur réputation au sein de cette arène.

${ }^{6}$. Alex Vines et Tom Cargill, «Le monde doit nous juger sur l'Afrique. L'héritage africain de Tony Blair », Politique africaine, $\mathrm{n}^{\circ} 101$, mars-avril 2006, p. 132-147.
} 
francophones et de certaines ONG notamment, et les contraintes imposées par les atermoiements de Washington sur ce dossier?

\section{Le leadership selon Washington : partager les responsabilités et limiter les risques de fiasco médiatique}

Le rôle particulier de la délégation américaine va cependant bien au-delà de cette idée de contrainte permissive. Tout au long des années 1990, sa capacité d'influence lui a permis de façonner le contenu politique du rôle de leader, et plus généralement de peser sur les choix de l'ONU en matière de maintien de la paix. Cette influence s'est notamment traduite par le renforcement de la position du leader au sein du Conseil et l'accroissement des responsabilités ouvertement assumées par lui dans la conduite d'une opération de maintien de la paix.

Après l'échec américano-onusien en Somalie, la diplomatie américaine a distillé un climat d'autocensure et de paralysie au sein du Conseil de sécurité en matière d'opérations de paix, en particulier en Afrique. On le sait, cela a joué un rôle considérable dans l'inaction de l'ONU pendant le génocide rwandais ${ }^{8}$. Cette autocensure a tenu en grande partie au souci du Département d'État - conscient de l'utilité de l'ONU et de ses opérations de paix pour les États-Unis - d'éviter toute nouvelle situation de fiasco pouvant être utilisée par les nombreux et influents pourfendeurs de l'ONU au sein même des États-Unis. L'objectif était de montrer à ces derniers, dominants au Congrès depuis novembre 1994, que les États-Unis ne pouvaient être considérés comme les seuls responsables des choix de l'ONU dans le maintien de la paix, et qu'ils n'appuyaient désormais que les opérations offrant les plus grandes garanties de succès. Et précisément, une de ces garanties envisagées fut bien de désigner publiquement une délégation comme responsable de l'action de l'ONU sur un terrain de conflit, et de confier à cette délégation la charge de mobiliser les soutiens diplomatiques et financiers suffisants en suscitant l'intérêt des donateurs et des médias.

Les diplomaties intéressées par le maintien de la paix et les responsables du Secrétariat de l'ONU ont alors dû céder à cette pression américaine. En 2000, Kofi Annan réussit ainsi à renégocier à la baisse (de 30 à $27 \%$ ) la quote-part des États-Unis dans le budget des opérations de paix de l'ONU. Par ailleurs, dans son important rapport pour la réforme des opérations de paix de l'ONU, le groupe d'étude présidé par Lakhdar Brahimi a insisté sur la nécessaire désignation d'un État leader au cœur des opérations de paix, d'un État capable de leur assurer la robustesse nécessaire, notamment militaire ${ }^{9}$.

Il incombe désormais au leader d'assurer, si nécessaire, la protection armée de l'opération de paix qu'il pilote face à tout risque d'échec médiatisé, de faiblesse visible sur le terrain, de retrait honteux ${ }^{10}$. Le choix de Londres d'engager ses troupes en 2000 en renfort de la

\footnotetext{
7. John B. Hay. Conditions of Influence: a Canadian Case Study in the Diplomacy of Intervention, Ottawa, Carleton University, The Norman Paterson School of International Affairs, occasional paper n 19, 1999.

8. Samantha Power, A Problem from Hell. America and the Age of Genocide, New York, Basic Books, 2002.

9. Rapport du Groupe d'étude sur les opérations de paix de l'ONU (dit rapport Brahimi), 17 août 2000 , A/55/305-S/2000/809, p. 5, § 25 .

10. David Ambrosetti, «Les opérations de paix de l'ONU face au risque d'un discrédit irrémédiable : la faiblesse et la sélectivité », Studia Diplomatica, vol. 59, $\mathrm{n}^{\circ}$ 2, 2006, p. 123-139. Cette importance de l'existence d'un État déterminé à fournir les ressources suffisantes et même à risquer des troupes pour la défense d'une opération de maintien de la paix face à ceux qui la défient, face au risque d'impuissance et d'un retrait honteux, est d'ailleurs une « recette » soulignée dans les ouvrages américains les plus influents en matière de résolution des conflits armés. Voir par exemple Stephen J. Stedman, Donald Rothchild et
} 
mission de l'ONU en Sierra Leone, à un moment où celle-ci était victime d'attaques des rebelles du Revolutionary United Front et menacée d'un retrait honteux sous pressions américaines, a montré le fort soutien qu'une telle initiative rencontrait dans la diplomatie américaine, ainsi que le prestige qu'elle a généré pour la diplomatie britannique dans le rang des délégations attachées au maintien de la paix de l'ONU. De même, la reconquête prudente d'un leadership par l'ambassadeur français à l'ONU, Jean-David Levitte, sur le conflit en République démocratique du Congo (RDC) à partir de 2001 a conduit les Français à déployer, sur le même modèle, une force militaire en RDC au printemps 2003 (opération Artémis), sous l'égide de l'Union européenne cette fois, pour pallier les carences de la mission de l'ONU (la Monuc) sur le terrain. Et d'une certaine manière, après bien des réticences, les États-Unis sont restés dans ce modèle de leadership lorsqu'ils ont soutenu le déploiement de casques bleus au Liberia en 2004 après le départ de Charles Taylor du pouvoir, et ont envoyé des soldats au Liberia à cette date, des soldats rapidement remplacés par des forces ouest-africaines, néanmoins. Au Liberia, comme en RDC ou au Soudan, cet engagement américain est apparu comme une façon de favoriser les intérêts des États-Unis dans ces régions tout en proposant une contrepartie à leur unilatéralisme de l'après 11Septembre et à la crise diplomatique autour de l'invasion armée de Irak en 2003.

Le renforcement de la position de leader s'est donc traduit par un accroissement des responsabilités et une visibilité accrue de ce rôle, avec pour résultat des opérations globalement moins exposées au risque de fiasco, mais également une sélectivité plus grande. Le leadership requiert un niveau de ressources et un degré d'engagement tel qu'il exclut bien des délégations, et bien des situations de crises présentant peu d'intérêts pour les quelques acteurs capables de l'assumer.

Le contenu donné au rôle de leader par les membres du Conseil de sécurité a fortement marqué le maintien de la paix à l'ONU et son évolution depuis la fin de la guerre froide. À travers les règles, informelles et changeantes, qui président à la reconnaissance collective de ce rôle se joue l'influence des diplomaties dans ce domaine d'action fortement investi par les États membres, en particulier par les États occidentaux. Sont également en jeu les modalités concrètes de réalisation des opérations de paix et les facteurs de leur succès ou de leur échec au plan opérationnel. Et en la matière, il semble bien que, en vertu de règles devenues très sélectives, les membres permanents du Conseil demeurent les mieux placés pour assurer ce leadership diplomatique au sein du Conseil, qui peut se transformer à l'occasion en interventionnisme armé. Toutefois, une fois décidées dans ce contexte de forte politisation du choix d'intervenir, ces opérations gagnent davantage de robustesse et de chances de succès opérationnel. En vertu de la reconnaissance qui lui est accordée, l'État leader s'engage en effet à constituer la colonne vertébrale ${ }^{11}$ de la mission, tant en termes matériels et logistiques (appuis militaires, transports de troupes) que diplomatiques (mobilisation de ses relais sur le terrain et dans les instances multilatérales, en particulier parmi les bailleurs de fonds internationaux). Les difficultés récemment rencontrées par la Monuc en République démocratique du Congo conduisent néanmoins à relativiser la portée

Elizabeth M. Cousens, Ending Civil Wars. The Implementation of Peace Agreements, International Peace Academy / Center for International Security and Cooperation, Boulder, Lynne Rienner, 2002, p. 3.

${ }^{11}$. Le terme de «backbone nation » revient ainsi fréquemment dans la bouche des diplomates, pour insister sur les responsabilités particulières du leader dans le montage et le suivi de l'opération de paix. 
de ces changements. Plus qu'une garantie de succès sur le terrain, ces changements assurent à tout le moins une réactivité du Conseil et de son leader sur un dossier donné en cas de forte mobilisation médiatique autour d'un échec annoncé de l'ONU. Avec un objectif politique prépondérant: éviter une nouvelle campagne anti-onusienne à Washington et dans les médias américains influents.

Cette évolution majeure est le fruit d'une décennie d'expérimentations onusiennes qui se reflètent dans le contenu politique donné à cette figure du leader. Résultat de l'ambivalence des États membres vis-à-vis du maintien de la paix, et en particulier celle des États-Unis, le rôle de leader ne peut se comprendre comme une traduction mécanique de l'échelle des puissances sur la scène internationale. Il s'inscrit dans un façonnement multilatéral des attentes, des responsabilités, des prérogatives, des critères de crédibilité, où le rapport de force n'est jamais absent mais se joue d'une façon très différente des voies unilatérales plus traditionnelles. 